\title{
Surface Polarons Reducing Overpotentials in the Oxygen Evolution Reaction
}

\author{
Patrick Gono, ${ }^{*}$ Julia Wiktor, Francesco Ambrosio, and Alfredo Pasquarello \\ Chaire de Simulation à l'Echelle Atomique (CSEA), Ecole Polytechnique Fédérale de \\ Lausanne (EPFL), CH-1015 Lausanne, Switzerland \\ E-mail: patrick.gono@epfl.ch
}

\section{Abstract}

We investigate the stability of hole polarons at the rutile surface induced by electronegative adsorbates in the intermediate steps of the oxygen evolution reaction through hybrid density functional calculations. Applying the computational hydrogen electrode method, we find that hole polarons reduce the overpotential of the reaction determining step leading to good agreement with experiment. The stability of the polarons is confirmed at the hydrated surface through a free energy study involving the explicit solvent. The occurrence of surface hole polarons is unrelated to the scaling relationships and offers an additional handle in the search for improved catalysts.

\section{Keywords}

surface hole polarons, oxygen evolution reaction, overpotential, oxygen dimer, rutile, $\mathrm{TiO}_{2}$

Efficient photocatalytic water splitting remains elusive even after decades of research.? The process can be split into two simultaneous reactions: the hydrogen evolution reaction, which takes place at the cathode, and the oxygen evolution reaction (OER) at the anode. While the former routinely reaches very high efficiencies, ${ }^{\text {? }}$ water splitting is limited by the kinetics of the latter. " Various restrictions apply to the catalyst in order to facilitate the OER, most notably the size of the band gap, the band alignment with respect to the redox levels, and the chemical stability in aqueous environment.? ? ?

From a theoretical point of view, a common approach to describe the reaction is the computational hydrogen electrode (CHE) method developed by Norskøv, Rossmeisl et al. ? It allows one to calculate the Gibbs free energy differences corresponding to the reaction intermediates. The OER is split into four steps, each involving a proton-coupled electron transfer:

$$
\begin{aligned}
*+\mathrm{H}_{2} \mathrm{O} & \longrightarrow \mathrm{OH}_{\mathrm{ads}}+\mathrm{H}^{+}+e^{-}, \\
\mathrm{OH}_{\mathrm{ads}} & \longrightarrow \mathrm{O}_{\mathrm{ads}}+\mathrm{H}^{+}+e^{-}, \\
\mathrm{O}_{\mathrm{ads}}+\mathrm{H}_{2} \mathrm{O} & \longrightarrow \mathrm{OOH}_{\mathrm{ads}}+\mathrm{H}^{+}+e^{-}, \\
\mathrm{OOH}_{\mathrm{ads}} & \longrightarrow{ }^{*}+\mathrm{O}_{2 \mathrm{~g}}+\mathrm{H}^{+}+e^{-},
\end{aligned}
$$

where the asterisk $\left(^{*}\right)$ refers to an active surface site. High-throughput screenings of potential catalysts have identified an interdependence of the free energy differences corresponding to the above reaction steps. ? ? This is referred to as the linear scaling relationship, which determines a restriction on the maximum efficiency of possible catalysts. ?

Titanium dioxide has been found to be a promising OER catalyst,? attracting a vast body of research.? ? ? ? ? Rutile $\mathrm{TiO}_{2}$ (r$\mathrm{TiO}_{2}$ ), and especially its (110) facet, has been extensively studied as a benchmark system to understand the reaction. ? ? ? ? ? ? ? ? Using the CHE approach at the semilocal level of density functional theory (DFT), the first step of Eq. (1) was found to be the reaction determining step with an overpotential of 
$0.8 \mathrm{eV}$. ? Cheng et al. calculated a similar value for this step using the thermodynamic integration method at the same level of theory. ? However, the application of hybrid functionals, a more advanced electronic structure method, results in an overpotential for the first step that is too high to be in accord with experiment.

The mechanism behind the OER at the rutile surface is assumed to be as follows: ? a photon is captured in the material and an electronhole pair is created consequently. The electron is conducted away from the anode. It has been shown that the holes form polarons that travel to the surface. ? The trapped hole then drives water dissociation, the initial step of the OER.? Hole trapping (i.e. localization of hole states) in $\mathrm{TiO}_{2}$ has been studied previously. ? ? ? ? ? Wang et al. ${ }^{\text {? }}$ considered that $\mathrm{OH}$ species adsorbed on the surface were at the origin of hole generation. The $\mathrm{OH}$ covered surface is one of the intermediates in the protoncoupled electron transfer model of the OER. Strongly electronegative species such as these may take an electron from the slab, thereby effectively generating a hole. In turn, the hole could then lead to the formation of a polaron at the surface.

In this Communication, we study the stability of surface hole polarons in the intermediates of the OER using hybrid functional theory. We assess the effect of these polarons on the free energies calculated via the computational hydrogen electrode method. Furthermore, we investigate the stability of these polarons at the hydrated surface adopting a free energy analysis and an explicit solvent.

We first study the stability of polarons at $\mathrm{OH}, \mathrm{O}$, and $\mathrm{OOH}$ covered surfaces in vacuum. The simulation cells contain five layer slabs of the $4 \times 2$ (110) rutile surface, with adsorbates on the fivefold coordinated titanium atoms on both sides of the slab, ${ }^{?}$ and a coverage of 0.25 . We model the proton-coupled electron transfer steps through intermediates in globally neutral charge states. The use of symmetric configurations prevents the build-up of electric fields in the simulation cell. A hybrid functional, which partially corrects the self-interaction error, is employed to better describe the local- ization of the polaronic states. ? ? The hybrid functional simulations are carried out with the PBE0? functional, in which the fraction of Fock exchange is set to $16 \%$, in order to reproduce the experimental band gap of $3.0 \mathrm{eV}$ of r- $\mathrm{TiO}_{2} .{ }^{\text {? }}$ This setting is supported by a recent study in which it is shown that a hybrid functional reproducing the correct band gap generally also gives an accurate energetic description of polarons. ?

Surface polarons consisting of $\mathrm{O}$ dimers are created by manually moving a surface plane oxygen and a neighboring bridge oxygen to a relative distance of $1.46 \AA$. Each polaron draws two positive charges, effectively leaving the adsorbates in a negatively charged state. The thermodynamic stability of the O-O dimer in the +2 charge state stems from the fact that this state is composed of two threefold coordinated $\mathrm{O}$ atoms in the charge state +1 , which satisfy the octet rule. Hole O-O bipolarons have already been proposed in various bulk transition metal oxides, such as anatase $\mathrm{TiO}_{2}, \mathrm{~V}_{2} \mathrm{O}_{5}$, and $\mathrm{MoO}_{3},{ }^{\text {? }}$ but also in amorphous $\mathrm{Al}_{2} \mathrm{O}_{3}$. ? In our calculations, two polarons are created on each side of the slab in a symmetric manner. A detailed description of the DFT geometry optimization can be found in the Supporting Information (SI). For comparison, we also consider the case of ${ }^{\bullet} \mathrm{OH}$ radicals on the surface. This can be realized by starting the relaxation from a configuration in which the Ti-O bonds between the surface and the adsorbates have slightly been elongated following ref. ? . However, we find that this configuration is higher in energy by $0.29 \mathrm{eV}$ per unit of trapped charge with respect to the O-O dimer polaron configuration. This implies that our hybrid functional scheme favors the occurrence of $\mathrm{OH}^{-}$rather than ${ }^{\bullet} \mathrm{OH}$. In comparison, the experimental characterization has so far been inconclusive in determining the relevant reaction intermediates, ${ }^{\text {? ? ? }}$ even though $\bullet \mathrm{OH}$ have been observed upon illumination.?

Figure 1 illustrates the relaxed surfaces with and without polarons. The figure also gives the DFT energy difference between the slab with polarons and the corresponding structure without polarons for the three intermediates, i.e. 
with $\mathrm{OH}, \mathrm{O}$, and $\mathrm{OOH}$ adsorbates. For both the $\mathrm{OH}$ and $\mathrm{OOH}$ covered surfaces, the structures with polarons are energetically more favorable, by as much as $1.58 \mathrm{eV}$ per polaron in the case of $\mathrm{OH}_{\text {ads }}$. For the oxygen covered surface, the adsorbed oxygen atom itself relaxes towards a position in which it forms a dimer with a surface plane oxygen.

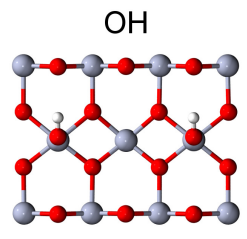

$-1.58 \mathrm{eV}$

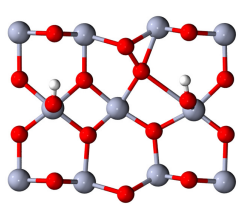

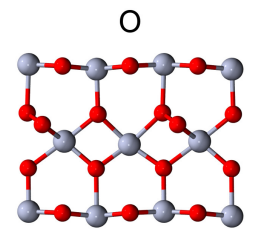

$0.73 \mathrm{eV}$

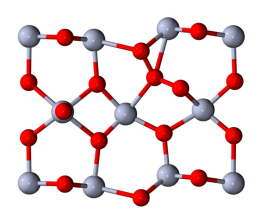

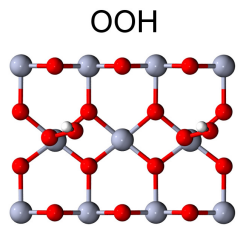

$-0.74 \mathrm{eV}$

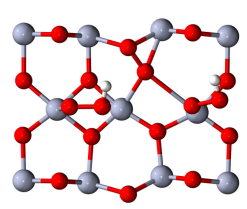

Figure 1: Top view of the relaxed surface layer of the OER intermediates without polarons (top) and with polarons (bottom). Ti atoms are shown in gray, $\mathrm{H}$ atoms in white, and $\mathrm{O}$ atoms in red. The total energy differences per polaron $\left(\Delta E_{\mathrm{pol}}\right)$ of the $\mathrm{OH}, \mathrm{O}$, and $\mathrm{OOH}$ covered slabs with and without polarons are shown. A negative sign indicates that the polaron formation leads to a lower total energy.

To understand the effect of polaron formation on the electronic structure, we study projected densities of states (PDOS) for the relaxed structures with and without polarons. Figure 2 shows the density of states of the $\mathrm{OH}$ covered slab projected on surface $\mathrm{O}$ atoms. In the absence of the polarons, we observe unoccupied levels inside the band gap localized on surface plane oxygen atoms, as well as on subsurface oxygen atoms. Upon the formation of the polarons, a sharp peak appears below the Fermi level (occupied levels). Likewise, we observe a relative increase in the PDOS at the bottom of the conduction band. The unoccupied states in the gap vanish. Hence, the creation of polarons stabilizes the system and pushes the unoccupied levels into the conduction band. A similar behavior is observed for the $\mathrm{OOH}$ covered surface.

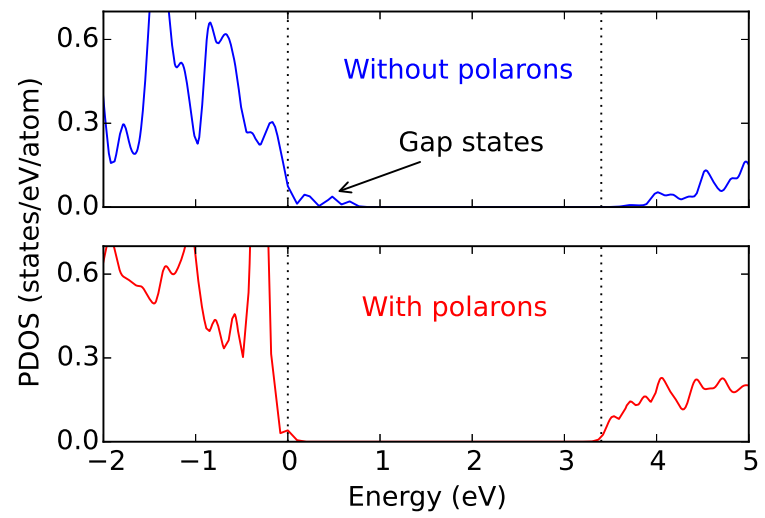

Figure 2: Projected density of states (PDOS) calculated for the $\mathrm{OH}$ covered rutile slab without (blue) and with (red) polarons. The density is projected on surface plane oxygen atoms. The projected densities are normalized with respect to the number of surface oxygen atoms. The energies are referred to the highest occupied state.

By taking into account surface polarons, we can quantify their effect on the energetics of the oxygen evolution reaction. Using the CHE approach, we calculate the free energy differences, $\Delta G_{1}$ through $\Delta G_{4}$, corresponding to the four proton-coupled electron transfer steps of water splitting given in Eq. (1). A detailed description of the method can be found in the Supporting Information. Our results are given in Table 1 and are illustrated in Figure 3. Nozik et al. estimated the experimental overpotential of the OER on $\mathrm{TiO}_{2}$ to lie between 0.9 and $1.1 \mathrm{eV}$ ? As seen in the work of Valdés et al., ${ }^{?}$ calculations based on the RPBE functional ${ }^{\text {? }}$ result in overpotentials that agree with the experimental findings. Our results at the RPBE level are consistent with this previous work, ${ }^{?}$ demonstrating the reliability of our setup. Next, we recalculate the free energies at the more advanced PBE0 level. As with the RPBE functional, the first step of the OER is the reaction determining step. With the PBE0 functional, the overpotential of this step increases to $1.52 \mathrm{eV}$, well above the experimental range $(0.9-1.1 \mathrm{eV}){ }^{?}$ A similar discrepancy between measured and hybridfunctional overpotentials had been found previously and was attributed to localized charges associated with unidentified defects. ? Our cal- 
culations demonstrate that surface polaron formation indeed reduces the overpotential of the reaction determining step to $0.73 \mathrm{eV}$, bringing the calculated result back in agreement with experimental observations.

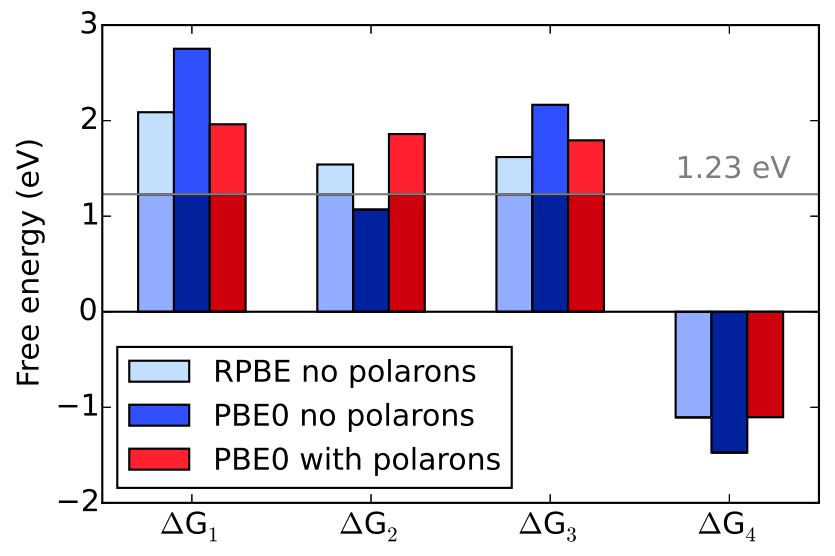

Figure 3: Gibbs free energy steps corresponding to the intermediates of the OER on $\mathrm{r}-\mathrm{TiO}_{2}$. The gray line indicates the standard potential of water splitting.

We now address the stability of surface polarons at the hydrated surface. To this end, we first carry out two molecular dynamics (MD) simulations at the hybrid functional level with explicit water solvent starting from different initial configurations with the $\mathrm{OH}$ adsorbate. In the first one the structure does not show any polaron, whereas four of them are present in the second one. More simulation details are given in the SI. Although the total energy of the structure with polarons is lower by about 1 $\mathrm{eV}$ per polaron, we do not observe spontaneous polaron formation over a MD duration of $3.5 \mathrm{ps}$ (see SI).

To properly assess the relative stability of the two structures, we hence perform a free energy analysis via the Blue Moon sampling scheme.

We carry out a series of constrained MD simulations, in which the distance between two relevant surface oxygen atoms is varied. The reaction coordinate $\xi$ thus corresponds to an $\mathrm{O}-\mathrm{O}$ distance and the Lagrange multipliers $\lambda$ associated with the constraint can be directly identified with the forces. ${ }^{?}$ The averages of the forces are used to calculate the free energy curve via?

$$
\Delta F=\int_{\xi_{0}}^{\xi_{1}} \mathrm{~d} \xi\langle\lambda\rangle_{\xi},
$$

where the reaction coordinate varies from $\xi_{0}=$ $2.75 \AA$ for the pristine structure to $\xi_{1}=1.46 \AA$ corresponding to polaron formation. We perform MD runs for seven values of the constraint. A duration of $0.75 \mathrm{ps}$ is sufficient for each of these runs to reach convergence. Figure 4 shows the free energy curve obtained from the integral of the average forces (see Figure S2 in the SI). The free energy of the structure with the polaron is indeed lower, following the same trend observed for the total energies. We find a free energy difference of $1.29 \pm 0.04 \mathrm{eV}$, to be compared with the value of $1.58 \mathrm{eV}$ obtained above for the surface in vacuum within the CHE approach. The effect of the water solvent can thus be estimated to be about $0.3 \mathrm{eV}$. The calculated free energy profile also reveals that there is a barrier of $0.21 \pm 0.03 \mathrm{eV}$ for polaron formation at the hydrated surface. This free energy barrier makes spontaneous polaron formation improbable in MD evolutions lasting only a few picoseconds, consistent with our observations. However, this barrier is sufficiently low to allow for the thermal generation of surface polarons at room temperature.

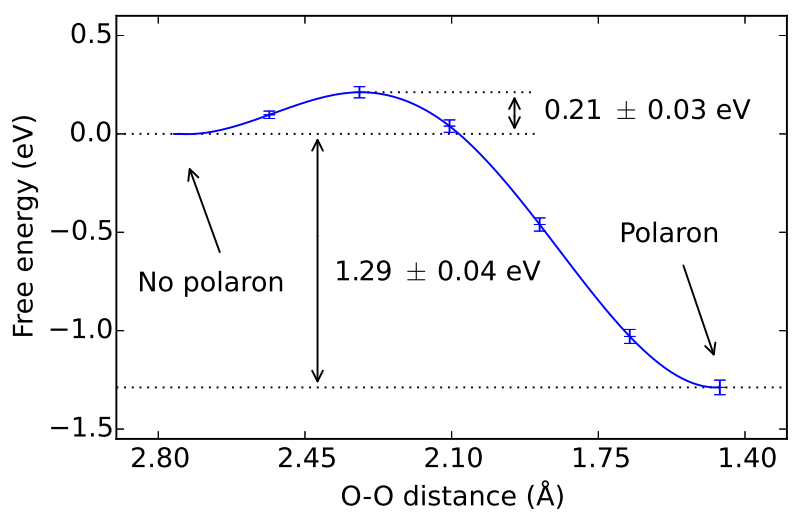

Figure 4: The free energy profile calculated by integrating the average forces for various fixed $\mathrm{O}-\mathrm{O}$ distances.

In summary, we demonstrated that the intermediates of the oxygen evolution reaction stabilize hole polarons at the rutile $\mathrm{TiO}_{2}$ surface. When polarons are considered, the calculated 
Table 1: Free energy differences corresponding to the four steps of the OER. Energies are given per active site and are in $\mathrm{eV}$.

\begin{tabular}{lcccccc} 
& Functional & Polarons & $\Delta G_{1}$ & $\Delta G_{2}$ & $\Delta G_{3}$ & $\Delta G_{4}$ \\
\hline Previous (Ref. ? ) & RPBE & No & 2.20 & 1.47 & 1.55 & -0.30 \\
Present & RPBE & No & 2.09 & 1.31 & 1.63 & -0.18 \\
Present & PBE0 & No & 2.75 & 1.01 & 2.22 & -1.46 \\
Present & PBE0 & Yes & 1.96 & 1.80 & 1.84 & -1.09 \\
\hline
\end{tabular}

overpotential of the reaction determining step is found to be lowered and to agree with experiment. The occurrence of polarons is material specific and affects the binding energies of the adsorbates. This effect is not considered in the linear scaling relationships, which limit the efficiency of the oxygen evolution reaction. Hence, polaron formation could partially overcome such limitations and should be systematically addressed in materials searches for the optimal catalyst.

\section{Supporting Information Available}

Computational parameters of the setups, description of the computational hydrogen electrode method and details on the study of the hydrated $\mathrm{TiO}_{2}$ surface

Acknowledgement We thank A. Bouzid and Z. Guo for useful interactions. This work has been realized in relation to the National Center of Competence in Research (NCCR) "Materials' Revolution: Computational Design and Discovery of Novel Materials (MARVEL)" of the SNSF. We used computational resources of the Swiss National Supercomputing Centre (CSCS) and of the Scientific IT and Application Support Center (SCITAS) of Ecole Polytechnique Fédérale de Lausanne. 


\section{Graphical TOC Entry}

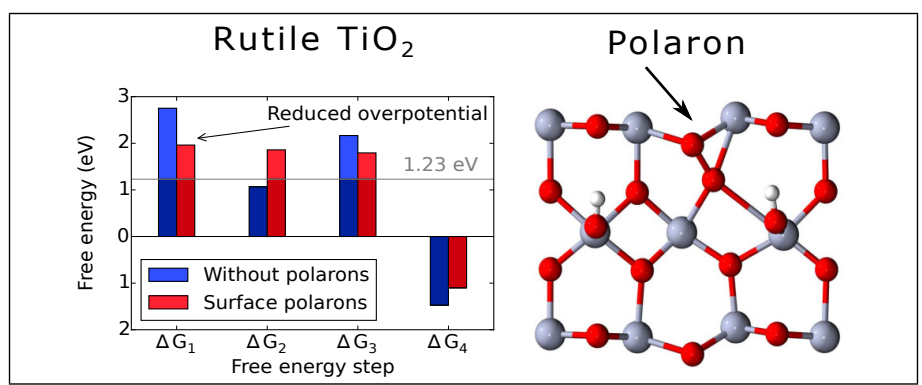

The effect of surface hole polarons on the overpotential of the oxygen evolution reaction on rutile $\mathrm{TiO}_{2}$. 